\title{
A very general binomial matrix
}

\section{Leo Betthauser ${ }^{1}$, Ömür Deveci ${ }^{2}$ and Josh Hiller ${ }^{3}$}

\author{
${ }^{1}$ Department of Mathematics, PO Box 118105, University of Florida \\ Gainesville, FL 32611-8105, United States \\ e-mail: lbetthauser@ufl.edu \\ ${ }^{2}$ Department of Mathematics, Faculty of Science and Letters, Kafkas University \\ 36100, Turkey \\ e-mail: odeveci36@hotmail.com \\ ${ }^{3}$ Department of Mathematics and Computer Science, Adelphi University \\ New York, United States \\ e-mail: johillerdadelphi.edu
}

\begin{abstract}
We define the very general binomial matrix and find its eigendecomposition over arbitrary rings when such a decomposition is possible. Using this decomposition, we are able to compute the order of several varieties of Pascal's matrices.
\end{abstract}

Keywords: Pascal's matrix, Binomial coefficients, Matrix.

2010 Mathematics Subject Classification: 11B65, 15A09, 15A16.

\section{Introduction}

The authors share a secret which we shall now make public, a secret which, we believe, many others will sympathize with: we were lured, almost tricked, into math by Pascal's triangle! The magical simplicity of its recursive formulation coupled with the myriad of deep identities which it holds, hidden within its entries, was just too much to resist as curious children.

Even now as adults and professional mathematicians this mysterious beauty continues to appear before us. In this we are not alone; the combinatorial masterpiece, in matrix form, has been found to arise naturally in theoretical probability [6], the study of general linear groups [11], image processing [2], and for the authors, quite unexpectedly, in the study of cancer. 
A very simple model of carcinogenesis (or the process of cancer formation in the body) is the so-called multi-hit model of Nordling (see the original paper [18] and [12] for a historical overview of related models). In this model, cancer is the final result of $n$ precancerous mutations. Thus if we assume that these mutations can happen in any order, and all mutations have a constant (and equal) transition rate $h>0$ then assuming a particular value for $n$, say 5 , we obtain a finite Markov chain with the following infinitesimal generator matrix:

$$
A=\left(\begin{array}{cccccc}
0 & 0 & 0 & 0 & 0 & 0 \\
h & -h & 0 & 0 & 0 & 0 \\
0 & 2 h & -2 h & 0 & 0 & 0 \\
0 & 0 & 3 h & -3 h & 0 & 0 \\
0 & 0 & 0 & 4 h & -4 h & 0 \\
0 & 0 & 0 & 0 & 5 h & -5 h
\end{array}\right) .
$$

Unfortunately, this matrix by itself is not really what concerns us. Instead, we required the time based transition probability matrix $P(t)$. Luckily, moving from our matrix $A$ to $P(t)$ is a fairly simple process and involves only taking the matrix exponential. Since these two matrices obey the relationship

$$
P(t)=e^{(t A)},
$$

where when $M$ is a square matrix,

$$
e^{M}=\sum_{i=0}^{\infty} \frac{M^{i}}{i !}
$$

is the matrix exponential function.

To make the matter even simpler, since $A$ is lower diagonal with no repeated entries along the diagonal then $A=L D L^{-1}$ where $L$ is some invertable matrix, and $D$ is the matrix consisting of the eigenvalues of $A$. From here we see that $t A=L(t D) L^{-1}$ and so

$$
P(t)=L\left[e^{(t D)}\right] L^{-1}
$$

since the values of $D$ can be read off directly from $A$ all that was needed was to calculate $L$. And here is where the ever present triangle reared its head: it can easily be verified that we can chose $L$ so that

$$
L=\left(\begin{array}{cccccc}
1 & 0 & 0 & 0 & 0 & 0 \\
1 & 1 & 0 & 0 & 0 & 0 \\
1 & 2 & 1 & 0 & 0 & 0 \\
1 & 3 & 3 & 1 & 0 & 0 \\
1 & 4 & 6 & 4 & 1 & 0 \\
1 & 5 & 10 & 10 & 5 & 1
\end{array}\right)
$$

and so Pascal has reared his head once again, with the Lower triangular Pascal matrix. After some reading, we realized that this was not the first time the lower triangular Pascal matrix has appeared as a change of basis matrix; in fact, the observation that this was a practical choice was discovered at least 10 years ago in the context of voting theory [15]! Perhaps just as interesting though, is that the lower triangular Pascal matrix is not unique in terms of utility. If we reorder the states of our cancer model we obtain the matrix 


$$
B=\left(\begin{array}{cccccc}
5 h & -5 h & 0 & 0 & 0 & 0 \\
0 & -4 h & 4 h & 0 & 0 & 0 \\
0 & 0 & -3 h & 3 h & 0 & 0 \\
0 & 0 & 0 & -2 h & 2 h & 0 \\
0 & 0 & 0 & 0 & -h & h \\
0 & 0 & 0 & 0 & 0 & 0
\end{array}\right)
$$

To diagonalize $B$, we can choose an involutory variation of the Pascal matrix for a change of basis matrix,

$$
V=V^{-1}=\left(\begin{array}{cccccc}
1 & -5 & 10 & -10 & 5 & -1 \\
0 & -1 & 4 & -6 & 4 & -1 \\
0 & 0 & 1 & -3 & 3 & -1 \\
0 & 0 & 0 & -1 & 2 & -1 \\
0 & 0 & 0 & 0 & 1 & -1 \\
0 & 0 & 0 & 0 & 0 & -1
\end{array}\right) .
$$

This matrix is similar, and bears a strong resemblance, to the involutory Pascal Matrix of Ashrafi and Gibson [2] which has applications in image processing and data description. This matrix has the form:

$$
W=W^{-1}=\left(\begin{array}{cccccc}
1 & -1 & 1 & -1 & 1 & -1 \\
0 & -1 & 2 & -3 & 4 & -5 \\
0 & 0 & 1 & -3 & 6 & -10 \\
0 & 0 & 0 & -1 & 4 & -10 \\
0 & 0 & 0 & 0 & 1 & -5 \\
0 & 0 & 0 & 0 & 0 & -1
\end{array}\right)
$$

While these three variations of the Pascal matrix are undoubtedly not the same, they are sufficiently alike to raise the question what else happens when we take some type of the Pascal matrix as a change of base matrix? What famous gems could we uncover? What open questions could we answer? Before attempting to explore these question we will give some background on some of the many famous and important variations of the Pascal matrix. Throughout this note, let us assume $n>1$ (no $1 \times 1$ matrices for us!) and $\mathbb{F}$ is a field.

\section{The fellowship of the Pascal matrices}

The history of the Pascal matrices dates back at least to the 1970's when Hogart and Bicknell explored several properties of the lower triangular Pascal's Matrix $L_{n}$ and used the inverses of its powers to derive combinatorial identities related to the Catalan numbers $[4,13,14]$. However, since the 1990s the study of these special matrices has taken flight. Hundreds of new properties have been brought to light through the combined efforts of dozens of researches from all the world (the reader interested in exploring some very beautiful general results is referred to [7,16,19-22]). As part of this journey of discovery, several variations on the idea of a Pascal matrix have been explored. What follows is a brief description of some of the more popular varieties. 


\subsection{The canonical trilogy}

How should we create an $n \times n$ Pascal's matrix? If one were to pose this question to most mathematicians, one would find in general three common answers:

- an upper triangular matrix $U_{n}$ with $(i, j)$-entry of $\left(U_{n}\right)_{i, j}=\left(\begin{array}{c}j-1 \\ i-1\end{array}\right)$,

- a lower triangular matrix $L_{n}$ with $(i, j)$-entry of $\left(L_{n}\right)_{i, j}=\left(\begin{array}{c}i-1 \\ j-1\end{array}\right)$,

- a symmetric matrix $S_{n}$ with $(i, j)$-entry of $\left(S_{n}\right)_{i, j}=\left(\begin{array}{c}i+j-2 \\ i-1\end{array}\right)$.

One of the most surprising results about these matrices is that they are all three linked through the following pleasing factorization [8]:

$$
L_{n} U_{n}=S_{n}
$$

Much of the research into variations of Pascal matrices can be traced back to the study of these three fundamental variations. For example, [11] used the $U_{n}$ matrix to find conjugate dense subgroups within the general linear group $G L_{n}(C)$, [1] defined their matrix as $L_{n}+I_{n}$ where $I_{n}$ is the $n \times n$ identity matrix and we can of course view $W_{n}$ as a variation of $U_{n}$.

$$
U_{4}=\left(\begin{array}{llll}
1 & 1 & 1 & 1 \\
0 & 1 & 2 & 3 \\
0 & 0 & 1 & 3 \\
0 & 0 & 0 & 1
\end{array}\right), L_{4}=\left(\begin{array}{cccc}
1 & 0 & 0 & 0 \\
1 & 1 & 0 & 0 \\
1 & 2 & 1 & 0 \\
1 & 3 & 3 & 1
\end{array}\right), \quad S_{4}=\left(\begin{array}{cccc}
1 & 1 & 1 & 1 \\
1 & 2 & 3 & 4 \\
1 & 3 & 6 & 10 \\
1 & 4 & 10 & 20
\end{array}\right)
$$

\subsection{Roads go ever on: three generalizations of the canonical trilogy}

Using the three matrices, $U_{n}, L_{n}$ and $S_{n}$ as seeds, mathematicians have formulated many generalizations of what a Pascal matrix looks like. From matrices which are functions in three variables over the reals [3] to changes of field from the real numbers (which is where many of the results came from) to finite fields and rings [9].

One important variation was put forth by Call and Velleman in 1993 [6] and independently in 1992 by [5]. This $n \times n$ matrix, which we will call $P_{1, n}(y)$ has as its $(i, j)$-entry

$$
\left(P_{1, n}\right)(y)=y^{j-i}\left(\begin{array}{c}
j-1 \\
i-1
\end{array}\right)
$$

and has become known as the generalized Pascal matrix of the first kind [9]. In the context of the real numbers, we find that this matrix arises quite naturally since for any real number $y$, the matrix power $U_{n}^{y}=P_{1, n}(y)$. However, once we leave the comfort of the real or complex numbers (which indeed we shall) it is still useful to have this matrix defined even though we no longer have the "power" interpretation available to us.

As an alternative to the power interpretation, Call and Velleman also established a very beautiful property for the field of real numbers: given the strictly upper triangular count matrix 


$$
C=\left(\begin{array}{ccccc}
0 & 1 & 0 & \ldots & 0 \\
0 & 0 & 2 & \ldots & 0 \\
\vdots & \vdots & \vdots & \ddots & \vdots \\
0 & 0 & 0 & \ldots & 0
\end{array}\right)
$$

Theorem 6 of [6] states that

$$
e^{z C}=P_{1, n}(z) .
$$

This immediately implies that $P_{1, n}^{-1}(z)=P_{1, n}(-z)$.

Not long after this well studied variation appeared in the literature, a new family of matrices was presented by Zhang [19], we shall call these matrices $P_{2, n}(x)$ where the $(i, j)$-entry is defined by:

$$
\left(P_{2, n}\right)_{i j}=x^{j+i}\left(\begin{array}{c}
j-1 \\
i-1
\end{array}\right) .
$$

We should say that when these generalized Pascal matrices of the second kind were introduced, the author operated under the convention that $P_{2, n}(0)=I$.

Both of these matrix families have been actively pursued in the literature. However, a third variation consists of a matrix function in two variables; it was introduced in 1998 and in fact gives us both $P_{1, n}(x)$ and $P_{2, n}(x)$ as special cases [17]. We denote this matrix $Q_{n}(y, x)$, and define its $(i, j)$-entry as

$$
(\rho)_{i j}=\left(y^{j-i}\right)\left(x^{j+i-2}\right)\left(\begin{array}{l}
j-1 \\
i-1
\end{array}\right)
$$

where $x$ is in the multiplicative group of a field, and $y$ is any element of the field. In the following theorem, we show how to diagonalize $Q_{n}(y, x)$. In this proof (and throughout the rest of this note), the matrix $D_{n}(z)$ is the $n \times n$ diagonal matrix with nonzero entry $z^{i-1}$ in the $i$-th row.

Theorem 1. Let $x, y \in \mathbb{F}$, and $x \notin\{-1,1,0\}$. Then $Q_{n}(x, y)$ can be factored as

$$
Q_{n}(y, x)=P_{1, n}(z) D_{n}\left(x^{2}\right) P_{1, n}(-z)
$$

where $z=\frac{y x}{x^{2}-1}$.

Proof. Since all the matrices in the product are upper triangular, we will concern ourselves only with the case when $j \geq i$. We denote the $i, j$ entry of $P_{1, n}(z)$ by $p_{i, j}$ and the $i, j$ entry of $P_{1, n}(-z)$ by $p_{i, j}^{\prime}$. Then, letting $b_{i, j}$ be the $i, j$ entry of the matrix $P_{1, n}(z) D\left(x^{2}\right) P_{1, n}(-z)$ we see that by defining

$$
a_{i, j}:=\sum_{k=1}^{n} d_{i, k} p_{k, j}^{\prime}=\left(x^{2}\right)^{i-1}\left(\frac{-x y}{x^{2}-1}\right)^{j-i}\left(\begin{array}{l}
j-1 \\
i-1
\end{array}\right)
$$

we can conclude that

$$
\begin{gathered}
b_{i, j}=\sum_{k=1}^{n} p_{i, k} a_{k, j}=\sum_{k=i}^{j}\left(\frac{x y}{x^{2}-1}\right)^{k-i}\left(\begin{array}{c}
k-1 \\
i-1
\end{array}\right)\left(x^{2}\right)^{k-1}\left(\frac{-x y}{x^{2}-1}\right)^{j-k}\left(\begin{array}{l}
j-1 \\
k-1
\end{array}\right) \\
=\sum_{k=i}^{j}(-1)^{j-k}\left(\frac{x y}{x^{2}-1}\right)^{j-i}\left(\begin{array}{c}
j-1 \\
k-1
\end{array}\right)\left(\begin{array}{c}
k-1 \\
i-1
\end{array}\right)\left(x^{2}\right)^{k-1}
\end{gathered}
$$




$$
\begin{gathered}
=\left(\frac{x y}{x^{2}-1}\right)^{j-i} \sum_{k=i}^{j}(-1)^{j-k}\left(\begin{array}{l}
j-1 \\
i-1
\end{array}\right)\left(\begin{array}{l}
j-i \\
k-i
\end{array}\right)\left(x^{2}\right)^{k-1} \\
=\left(\frac{x y}{x^{2}-1}\right)^{j-i}\left(\begin{array}{c}
j-1 \\
i-1
\end{array}\right) \sum_{k=i}^{j}(-1)^{j-k}\left(\begin{array}{l}
j-i \\
k-i
\end{array}\right)\left(x^{2}\right)^{k-1} \\
=\left(\frac{x y}{x^{2}-1}\right)^{j-i}\left(\begin{array}{c}
j-1 \\
i-1
\end{array}\right) \sum_{k=0}^{j-i}\left(\begin{array}{c}
j-i \\
k
\end{array}\right)(-1)^{j-i-k}\left(x^{2}\right)^{k+i-1} \\
=\left(\frac{x y}{x^{2}-1}\right)^{j-i}\left(\begin{array}{c}
j-1 \\
i-1
\end{array}\right)\left(x^{2}\right)^{i-1} \sum_{k=0}^{j-i}\left(\begin{array}{c}
j-i \\
k
\end{array}\right)(-1)^{j-i-k}\left(x^{2}\right)^{k} \\
=\left(\begin{array}{c}
x y \\
x^{2}-1
\end{array}\right)^{j-i}\left(\begin{array}{c}
j-1 \\
i-1
\end{array}\right)\left(x^{2}\right)^{i-1}\left(x^{2}-1\right)^{j-i} \\
=(x y)^{j-i}\left(\begin{array}{c}
j-1 \\
i-1
\end{array}\right) x^{2 i-2} \\
=\left(y^{j-i}\right)\left(x^{j+i-2}\right)\left(\begin{array}{c}
j-1 \\
i-1
\end{array}\right)
\end{gathered}
$$

proving that $b_{i, j}=\rho_{i, j}$.

We hope that the reader notices that this proof relies only on the binomial theorem (and none of the properties of the real or complex fields), and because of this fact we can derive the following corollary which will be useful later:

Corollary 2. Let $\mathbb{F}$ be a field, assume $x \notin\{-1,1,0\}$ and let $y \neq 0$. Then the order of $Q_{n}(x, y)$ is the order of $x^{2}$ in $\mathbb{F}^{\times}$(the multiplicative group of units of the field $\mathbb{F}$ ).

This is in fact a very nice generalization of a much more restrictive version of this result, which was first conjectured in 2012 for the case of $P_{1, n}(x)$ over the field $\mathbb{F}_{p}$, where $p$ is a prime. This conjecture was shown to be true in that very special case in 2014 [11]. However, the proof presented in the 2014 paper does not generalize to the case of $Q_{n}(x, y)$ nor does it hold for arbitrary fields.

\section{A (very) general binomial matrix}

It would seem from the introduction and Theorem 1 that the key to building some interesting matrices is to start with a diagonal matrix and transform it through a binomial choice of basis. And so we have found a new role for $P_{1, n}(x)$ within a more general setting. We no longer view it as a power of $U_{n}$ (which might not make sense over an arbitrary field), but as a useful basis choice for diagonal linear transformations. To see an example of the power of this view let us define the following very general binomial matrix $F_{n}(y, x)$ with $(i, j)$-entry given by: 


$$
\left(F_{n}\right)_{i j}=y^{j-i} x^{i-1}\left(\begin{array}{c}
j-1 \\
i-1
\end{array}\right) .
$$

Then $W_{n}=F_{n}(-1,1)$, and $Q_{n}(x, y)=F_{n}\left(x y, x^{2}\right)$ (so long as $y$ or $x$ is not 1 ). Because this family of matrices encompasses $Q_{n}(y, x)$ and $V_{n}$, it automatically generalizes $P_{1, n}(y)$ and $P_{2, n}(y)$. We now demonstrate how to diagonalize $F_{n}$ in the following theorem.

Theorem 3. Let $x, y \in \mathbb{F}$, such that $x \neq 0$ then:

$$
F_{n}(y, x)=P_{1, n}(z) D_{n}(x) P_{1, n}(-z),
$$

where $z=y /(x-1)$

The proof of this theorem is a near identical argument to that of Theorem 1, so we omit it here. The power in this theorem is that it is self-improving-it hints at the right hypothesis for further results! For example, by substituting $D\left(x^{-1}\right)$ in for $D(x)$ we can see that in the case where $F_{n}(y, x)$ is diagonalizable, we can find an explicit inverse. It turns out however, that the formula for this inverse works even in the case when $F_{n}(y, x)$ is not diagonalizable.

Theorem 4. For any values $y, x \in \mathbb{F}$, if $F_{n}(y, x)$ is invertable then

$$
F_{n}(y, x)^{-1}=F_{n}(-y / x, 1 / x) .
$$

The proof of this theorem is left as an exercise to the eager reader but involves only an application of the Binomial Theorem and matrix multiplication.

\section{Conclusion}

In this paper we gave a brief review of several popular variations of Pascal matrices and examined an expansive family of these matrices over arbitrary fields. Undoubtedly, however, the new variation presented here will not be the final variety of Pascal's treasure! These generalizations can be fantastic starting points for motivated undergraduates interested in number theory, probability, linear algebra, or even mathematical biology to start independent research projects. One observation, which we will leave to the interested reader, is that when we left this general setting and wandered back into the comfort of the real numbers, we were able to use our new variation to provide an unexpected proof that the alternating sum of binomial coefficients vanishes. This basically used only the matrix exponential (the binomial theorem remains hidden and is only used indirectly). We thus close this article by posing the question: What other gems might be uncovered with the help of Pascal's magical triangle in matrix form?

\section{Acknowledgements}

The authors are indebted to an anonymous referee for a careful reading, excellent suggestions, and for providing an even more general variation of Pascal's matrices than the one originally cooked up by the authors. 


\section{References}

[1] Aggarwala, R., \& Lamoureux, M. P. (2002). Inverting the Pascal matrix plus one. American Mathematical Monthly, 109(4), 371-377.

[2] Ashrafi, A., \& Gibbson, P. M. (2004). An involutory Pascal matrix. Linear Algebra and its Applications, 387, 277-286.

[3] Bayat, M., Asadi, M., \& Khatami, Z. (2014). The linear algebra of the right justified Pascal functional matrix with three variables. Caspian Journal of Applied Science Research, 3(1), $51-58$.

[4] Bicknell, M., \& Hoggatt, V. E. (1973). Unit determinants in generalized Pascal triangles. The Fibonacci Quarterly, 11(2), 131-144.

[5] Brawer, R., \& Pirovino, M. (1992). The linear algebra of the Pascal matrix. Linear Algebra and its Applications, 174, 13-23.

[6] Call, G. S., \& Velleman, D. J. (1993). Pascal's matrices. American Mathematical Monthly, 100(4), 372-376.

[7] Cronin, A. G., \& Laffey, T. J. (2015). On a conjecture of Deveci and Karaduman. Linear Algebra and its Applications, 471, 569-574.

[8] Edelman, A., \& Strang, G. (2004). Pascal matrices. American Mathematical Monthly, 111(3), 189-197.

[9] Deveci. Ö., \& Karaduman, E., (2012). The cyclic groups via the Pascal matrices and the generalized Pascal matrices. Linear Algebra and its Applications, 437(10), 2538-2545.

[10] Hiller, J., (2013). A proof of two conjectures of Deveci and Karaduman. Linear Algebra and its Applications, 446, 163-165.

[11] Hiller, J. (2016). Old friends in unexpected places: Pascal (and other) matrices in $G \operatorname{Ln}(\mathbb{C})$. American Mathematical Monthly, 123(2), 161-167.

[12] Hiller, J., Vallejo, C., Betthauser, L., \& Keesling, J. (2017). Characteristic patterns of cancer incidence: Epidemiological data, biological theories, and multistage models. Progress in Biophysics and Molecular Biology, 124, 41-48.

[13] Hoggatt, V. E., \& Bicknell, M. (1976). Pascal, Catalan, and general sequence convolution arrays in a matrix, The Fibonacci Quarterly, 14(2), 135-143.

[14] Hoggatt, V. E., \& Bicknell, M. (1976). Catalan and related sequences arising from inverses of Pascal's Triangle Matrices, The Fibonacci Quarterly, 14(5), 395-405.

[15] Jameson, M. K., Minton, G., \& Orrison, M. E. (2008). Borda Meets Pascal, Math Horizons, $16(1), 8-21$. 
[16] Lewis, B. (2010). Revisiting the Pascal matrix. American Mathematical Monthly, 117(1), $50-66$.

[17] Liu, M., \& Zhang, Z. (1998). An Extension of the Generalized Pascal Matrix and its Algebraic Properties. Linear Algebra and its Applications, 271, 169-177.

[18] Nording, C. (1951). A new theory on the cancer-inducing mechanism. British Journal of Cancer, 7(1), 68-72.

[19] Zhang, Z. (1997). The linear algebra of the generalized Pascal Matrix. Linear Algebra and its Applications, 250, 51-60.

[20] Zhang, Z., \& Liu, M. (1998). An extension of the generalized pascal matrix and its algebraic properties. Linear Algebra and its Applications, 271, 169-177.

[21] Wang, T., \& Zhang, Z. (1998). A Generalized Pascal matrix and recurrence sequences. Linear Algebra and its Applications, 283, 289-299.

[22] Zhang, Z., \& Wang, X. (2007). A factorization of the symmetric Pascal matrix involving the Fibonacci matrix. Discrete Applied Mathematics, 155, 2371-2376. 